\title{
Basis Existence of Internal n-Graph Space
}

\author{
Ahmad Lazwardi \\ University of Muhammadiyah Banjarmasin \\ Email: lazwardiahmad@gmail.com
}

\begin{abstract}
Graph of real valued continuous function with special addition and multiplication has already proven that is isomorphic to real number system. Furthermore, the graph of continous real valued function forms a field. The aim of this research was to generalize such concept to its n-tuple Cartesian Product and to prove that interchange of basis still able to be executed. The result of this research is n-tuple Cartesian Product of graph function forms a vector space over $\mathbb{R}$ and interchange of basis still able to be executed.
\end{abstract}

Keywords: n-Graph Space; General Vector Space; Internal n-Graph Space

\section{INTRODUCTION}

By associating the special operation to a graph of continuous function, such graph can be claimed as a vector space. This follows from the fact that every graph of continuous real valued function has a bijection to its domain i.e. the real number system. Furthermore, they are homeomorphic.

Graph of real valued continuous function has a unique characteristic. It has continuous shape of curve along real line $\mathbb{R}$. More detail will be described as follows [1]

Definition 1. Let real valued function $f: \mathbb{R} \rightarrow \mathbb{R}$. The graph of $f$ is defined as

$$
\mathbb{R}_{f}=\{(x, f(x)): x \in \mathbb{R}\}
$$

Continuity of $f$ indicates that $\mathbb{R}_{f}$ topologically equivalent to $\mathbb{R}$. Its described briefly as follows;

Definition 2 [2]. For each $U \subset \mathbb{R}$. Define the image of $U$ over $\mathbb{R}_{f}$ as

define the associated topology for $\mathbb{R}_{f}$ as

$$
U \times f(U)=\{(x, f(x)): x \in U\}
$$

$$
\tau_{f}=\{U \times f(U): U \text { open }\}
$$

It can be shown trivially that such topology implies $\mathbb{R}_{f}$ and $\mathbb{R}$ are homeomorphics . The fact that $\mathbb{R}_{f}$ and $\mathbb{R}$ are homeomorphics describes that even though their graph geometrically has different shapes, but they still have similarity in views of topology[3]. It motivates us to explore more special properties of $\mathbb{R}_{f}$.

Now let see it further.

Definition 3. Let real valued function $f: \mathbb{R} \rightarrow \mathbb{R}$. Define the addition for $\mathbb{R}_{f}$ as follow 
for any $x_{f}, y_{f} \in \mathbb{R}_{f}$.

$$
x_{f} \oplus y_{f}=(x+y, f(x+y))
$$

Define the scalar multiplication as follow

$$
\begin{gathered}
(\alpha) x_{f}=T_{f}\left(\alpha T_{f}^{-1}(x, f(x))\right) \\
=(\alpha x, f(\alpha x))
\end{gathered}
$$

For any $x_{f} \in \mathbb{R}_{f}$ and scalar $\alpha$, and $T_{f}$ is natural bijection generated by real valued function $f$ such as $T_{f}(x)=(x, f(x))$.

By associating $\mathbb{R}_{f}$ with those operations, $\mathbb{R}_{f}$ become real vector space. Moreover $\mathbb{R}_{f}$ and $\mathbb{R}$ are isomorphics [4].

\section{METHODS}

The method of this research is done by following method: first was to prove that $\mathbb{R}_{f}$ has dimension 1 and isomorphics to $\mathbb{R}$. Next step was to analyize more general space i.e. $n$ tuple Cartesian Product of $\mathbb{R}_{f}$ denoted by $\mathbb{R}_{f}^{n}$. In generalization of $\mathbb{R}_{f}^{n}$ was to prove that such space is a vector space over $\mathbb{R}$ and was able to change of bases. Finally, was to find out the briefly method to change of bases as well as in real vector spaces.

\section{RESULTS AND DISCUSSION}

It was proven previously that for each continuous function $f: \mathbb{R} \rightarrow \mathbb{R}$, the space $\mathbb{R}_{f}$ generated by $f$ forms a field. This fact becomes the basic to generalize the idea by constructing new n-tuple Cartesian Product of $\mathbb{R}_{f}$ which preserves vector space properties [5]. Before any further discussion, first define some necessary terms in order to help in generalization. We mean linear combination is $z_{f}=(\propto) x_{f}+(\beta) y_{f}$ for each $z_{f} \in$ $\mathbb{R}_{f}$ and some scalars $\alpha, \beta \in \mathbb{R}[6]$. The set of linear combinations of $x_{f}, y_{f}$ is named as Span $\left\{x_{f}, y_{f}\right\}$ [7]. The set $U \subset \mathbb{R}_{f}$ is said to be linearly independent if none of its members is able to expressed as linear combination of other members. Here is definition of basis:

Definition 4. The set $\left\{x_{f}^{1}, x_{f}^{2}, \ldots, x_{f}^{n}\right\}$ is called basis of subspace $U \subset \mathbb{R}_{f}$ if $\left\{x_{f}^{1}, x_{f}^{2}, \ldots, x_{f}^{n}\right\}$ are linearly independent and $\operatorname{Span}\left\{x_{f}^{1}, x_{f}^{2}, \ldots, x_{f}^{n}\right\}=U$.

Recall that, dimension of $U$ is defined as base cardinality of $U$.

Next theorem is an important result.

Theorem 1. $\mathbb{R}_{f}$ has dimension 1 .

Proof:

Chose $1_{f} \in \mathbb{R}_{f}$. For each $x_{f} \in \mathbb{R}_{f}$, it's obvious that

$$
\begin{aligned}
\mathrm{x}_{f} & =(x, f(x)) \\
& =T_{f}(x) \\
& =T_{f}(x .1) \\
& =(x .1, f(x .1)) . \\
& =(x) \times 1_{f}
\end{aligned}
$$

By last equation, we conclude that $\operatorname{Span}\left\{1_{f}\right\}=\mathbb{R}_{f}$.

Furthermore, based on the above results, vector space theory of $\mathbb{R}_{f}$ can be developed: 
Definition 5. Let $S=\left\{f_{1}, f_{2}, \ldots, f_{n}\right\}$ be a finite collection of real valued continuous functions, define $\mathbf{n}$-graph space as

$$
\mathbb{R}_{f_{p}}^{n}=\prod_{k=1}^{n} \mathbb{R}_{f_{k}}=\left\{\vec{v}:\{1,2, \ldots, n\} \rightarrow \bigcup_{k=1}^{n} \mathbb{R}_{f_{k}}: \vec{v}(i) \in \mathbb{R}_{f_{i}}\right\}
$$

Definition 6. Let $S=\left\{f_{1}, f_{2}, \ldots, f_{n}\right\}$ be a finite collection of real valued continuous functions. Define addition on $\mathbb{R}_{f_{p}}^{n}$ as

and scalar multiplication as

$$
\vec{v} \oplus \vec{w}=(\vec{v}(1) \oplus \vec{w}(1), \vec{v}(2) \oplus \vec{w}(2), \ldots, \vec{v}(n) \oplus \vec{w}(n))
$$

$$
(\propto) \vec{v}=((\propto) \vec{v}(1),(\propto) \vec{v}(2), \ldots,(\propto) \vec{v}(n))
$$

for each $\alpha \in \mathbb{R}$.

Theorem 2. Let $S=\left\{f_{1}, f_{2}, \ldots, f_{n}\right\}$ be a finite collection of real valued continuous function. n-graph space $\mathbb{R}_{f_{p}}^{n}$ is a vector space over $\mathbb{R}$ under operation $\oplus$ and scalar multiplication $(\propto) \vec{v}$.

In special case which $f_{1}=f_{2}=f_{3}=\cdots=f_{n}$, graph space $\mathbb{R}_{f_{p}}^{n}$ is called internal n-graph space i.e. Cartesian Product n-tuple of $\mathbb{R}_{f}$ itself. One can write

$$
\mathbb{R}_{f p}^{n}=\mathbb{R}_{f}^{n} \text {. }
$$

Euclidean Space $\mathbb{R}^{n}$ is one of finest example of graph space which $f$ is defined as identity mapping.

One of the most important tools to analyze relation between two internal n-graph space is linear transformation[8], here we still able to define linear transformation as well as done on commonly vector spaces.

Definition 7. Let two graph spaces $\mathbb{R}_{f}^{m}, \mathbb{R}_{f}^{n}$. Mapping $L: \mathbb{R}_{f}^{m} \rightarrow \mathbb{R}_{f}^{n}$ is linear transformation if the following properties hold

$$
\begin{array}{cc}
L(\vec{v} \oplus \vec{w})=L(\vec{v}) \oplus L(\vec{w}), \quad & \forall \vec{v}, \vec{w} \in \mathbb{R}_{f}^{m} \\
L((\propto) \vec{v})=(\propto) L(\vec{v}), & \forall \propto \in \mathbb{R}
\end{array}
$$

The set of all linear transformation from $\mathbb{R}_{f}^{m}$ to $\mathbb{R}_{f}^{n}$ is denoted as $\operatorname{Lin}\left(\mathbb{R}_{f}^{m}, \mathbb{R}_{f}^{n}\right)$ [7]. One of necessary example of linear transformation is linear mapping from $\mathbb{R}_{f}^{m}$ to its coordinate i.e.

by specific formula

$$
\overrightarrow{v_{l}}: \mathbb{R}_{f}^{m} \rightarrow \mathbb{R}
$$

$\overrightarrow{v_{l}}(\vec{v})=\vec{v}(i), \quad i=1,2,3, \ldots, m$.

The term of linear transformation is very useful in constructing theory change bases in graph space[9]. On internal graph space $\mathbb{R}_{f}^{m}$ change of bases still able to be constructed.

Definition 8. Let $\vec{v}_{1}, \vec{v}_{2}, \ldots, \vec{v}_{k} \in \mathbb{R}_{f}^{m}$ are said to be linearly independent if for each $\vec{v}_{l}$ can't be expressed as linear combination of some others.

The term of linear combination refers to equation

$$
\vec{v}=\left(\propto_{1}\right) \overrightarrow{v_{1}} \oplus \ldots \oplus\left(\propto_{k}\right) \overrightarrow{v_{k}}
$$

Another way to express coordinate transformation is by defining linear transformation which maps a vector to its scalars corresponding to the basis used to. 
Definition 9. Let $V$ be a nontrivial vector space over field $F$. Let $S=\left\{\overrightarrow{s_{1}}, \overrightarrow{s_{2}}, \overrightarrow{s_{3}}, \ldots, \overrightarrow{s_{m}}\right\}$ be a basis of $V$. Define coordinate transformation as $\emptyset_{S}: V \rightarrow F^{m}$ such that for each $\vec{v}=v_{1} \overrightarrow{s_{1}}+$ $v_{2} \overrightarrow{S_{2}}+\cdots+v_{m} \overrightarrow{S_{m}}$, the map

$$
\emptyset_{S}(\vec{v})=[\vec{v}]_{S}=\left[\begin{array}{c}
v_{1} \\
v_{2} \\
\vdots \\
v_{m}
\end{array}\right] \text {. }
$$

It's easy to check that coordinate transformation is an isomorphism [10]. The concept of coordinate mapping above is used to construct the concept of change of basis. First, we have to decide standard basis which lies on $\mathbb{R}_{f}^{m}$.

Standard basis of $\mathbb{R}_{f}^{m}$ is

$$
\begin{aligned}
& \overrightarrow{v_{l}}=\left(1_{f}, 0_{f}, 0_{f}, 0_{f}, \ldots, 0_{f}\right) \\
& \overrightarrow{v_{2}}=\left(0_{f}, 1_{f}, 0_{f}, 0_{f}, \ldots, 0_{f}\right) \\
& \overrightarrow{v_{m}}=\left(0_{f}, 0_{f}, 0_{f}, 0_{f}, \ldots, 1_{f}\right)
\end{aligned}
$$

Now let's see how the change of basis works:

For each $\vec{v} \in \mathbb{R}_{f}^{m}$. By previous definition, one can write

$$
\vec{v}=(\vec{v}(1), \vec{v}(2), \ldots, \vec{v}(m))
$$

which $\vec{v}(i) \in \mathbb{R}_{f}$ for each $i=1,2, \ldots, m$. Therefore

$$
\vec{v}=\vec{v}(1) \vec{v}_{1} \oplus \vec{v}(2) \vec{v}_{2} \oplus \ldots \oplus \vec{v}(m) \vec{v}_{m}
$$

for corresponding coordinate $\vec{v}(i) \in \mathbb{R}_{f}$.

This is how the above coordinate and corresponding scalars related, let's see it as follows:

Hence

$$
\begin{aligned}
\vec{v}(1) .1_{f} & =\vec{v}(1)=T_{f}\left(T_{f}^{-1}(\vec{v}(1))\right) \\
& =T_{f}\left(T_{f}^{-1}(\vec{v}(1) \cdot 1)\right. \\
& =\left(T_{f}^{-1}(\vec{v}(1) \cdot 1), f(\vec{v}(1) \cdot 1)\right) \\
& =\left(T_{f}^{-1}(\vec{v}(1) \cdot 1) \cdot 1_{f}\right.
\end{aligned}
$$

for some $\left(T_{f_{k}}^{-1}(\vec{v}(1)) \in \mathbb{R}\right.$.

$$
\vec{v}=\left(T _ { f } ^ { - 1 } ( \vec { v } ( 1 ) ) \cdot \vec { v _ { 1 } } \oplus \left(T _ { f } ^ { - 1 } ( \vec { v } ( 2 ) ) \cdot \vec { v _ { 2 } } \oplus \ldots \oplus \left(T_{f}^{-1}(\vec{v}(m)) \cdot \overrightarrow{v_{m}}\right.\right.\right.
$$

The last equation can be expressed in term of more visual vector addition as follows:

$$
\begin{aligned}
\vec{v} & =\left(T _ { f } ^ { - 1 } ( \vec { v } ( 1 ) ) \cdot \vec { v _ { 1 } } \oplus \ldots \oplus \left(T_{f}^{-1}(\vec{v}(m)) \cdot \overrightarrow{v_{m}}\right.\right. \\
& =\left(T _ { f } ^ { - 1 } ( \vec { v } ( 1 ) ) \cdot [ \begin{array} { c } 
{ 1 _ { f } } \\
{ 0 _ { f } } \\
{ \vdots } \\
{ 0 _ { f } }
\end{array} ] \oplus \ldots \oplus \left(T_{f}^{-1}(\vec{v}(m))\left[\begin{array}{c}
0_{f} \\
0_{f} \\
\vdots \\
1_{f}
\end{array}\right]\right.\right. \\
& =\left[\begin{array}{cccc}
1_{f} & 0_{f} & \ldots & 0_{f} \\
0_{f} & \ldots & \ldots & 0_{f} \\
\vdots & \ldots & \ldots & \vdots \\
0_{f} & 0_{f} & \ldots & 1_{f}
\end{array}\right]\left[\begin{array}{c}
T_{f}^{-1}(\vec{v}(1)) \\
\ldots \\
\ldots \\
T_{f}^{-1}(\vec{v}(m))
\end{array}\right]=I_{f} T_{f}^{-1}(\vec{v})
\end{aligned}
$$

The above equation gives a consequence that $\mathbb{R}_{f}^{m}$ is isomorphic to $\mathbb{R}^{m}$.

The next theorem will ensure that $\mathbb{R}_{f}^{m}$ has more than just standard basis.

Teorema 3. If $S=\left\{\boldsymbol{s}_{1}, \boldsymbol{s}_{2}, \ldots, \boldsymbol{s}_{m}\right\}$ is basis for $\mathbb{R}^{m}$ then $T_{f}(S)=\left\{T_{f}\left(\boldsymbol{s}_{1}\right), T_{f}\left(\boldsymbol{s}_{2}\right), \ldots, T_{f}\left(\boldsymbol{s}_{m}\right)\right\}$ forms a basis for $\mathbb{R}_{f}^{m}$.

Bukti: 
Suppose that $\vec{v} \in \mathbb{R}_{f}^{m}$. Let's see the following fact, another fine expression for vector in $\mathbb{R}_{f}^{m}$ is $\vec{v}=\left[\begin{array}{c}\vec{v}(1) \\ \vec{v}(2) \\ \vdots \\ \vec{v}(m)\end{array}\right]$. Thus we have $T_{f}^{-1}(\vec{v})=\left[\begin{array}{c}T_{f}^{-1}(\vec{v}(1)) \\ T_{f}^{-1}(\vec{v}(2)) \\ \vdots \\ T_{f}^{-1}(\vec{v}(m))\end{array}\right]$ is actually lies in $\mathbb{R}^{m}$. Therefore $T_{f}^{-1}(\vec{v})=\left[\begin{array}{c}T_{f}^{-1}(\vec{v}(1)) \\ T_{f}^{-1}(\vec{v}(2)) \\ \vdots \\ T_{f}^{-1}(\vec{v}(m))\end{array}\right]=\left(\propto_{1}\right)\left[\begin{array}{c}s_{11} \\ s_{12} \\ \vdots \\ s_{1 m}\end{array}\right]+\ldots+\left(\propto_{m}\right)\left[\begin{array}{c}s_{m 1} \\ s_{m 2} \\ \vdots \\ s_{m m}\end{array}\right]$, by $\boldsymbol{s}_{i}=\left[\begin{array}{c}s_{i 1} \\ s_{i 2} \\ \vdots \\ s_{i m}\end{array}\right]$ for each $i$

One can have $\vec{v}=T_{f}\left(T_{f}^{-1}(\vec{v})\right)=T_{f}\left(\left(\alpha_{1}\right) \boldsymbol{s}_{1}+\left(\alpha_{2}\right) \boldsymbol{s}_{2}+\ldots+\left(\alpha_{m}\right) \boldsymbol{s}_{m}\right)$

$$
\begin{aligned}
& =\left(\left(\alpha_{1}\right) \boldsymbol{s}_{1}+\left(\alpha_{2}\right) \boldsymbol{s}_{2}+\ldots+\left(\alpha_{m}\right) \boldsymbol{s}_{m}, f\left(\left(\alpha_{1}\right) \boldsymbol{s}_{1}+\left(\alpha_{2}\right) \boldsymbol{s}_{2}+\ldots+\left(\alpha_{m}\right) \boldsymbol{s}_{m}\right)\right) \\
& =\left(\alpha_{1}\right) T_{f}\left(\boldsymbol{s}_{1}\right) \oplus\left(\alpha_{2}\right) T_{f}\left(\boldsymbol{s}_{2}\right) \oplus \ldots \oplus\left(\alpha_{m}\right) T_{f}\left(\boldsymbol{s}_{m}\right) .
\end{aligned}
$$

In other words, $\left\{T_{f}\left(\boldsymbol{s}_{1}\right), T_{f}\left(\boldsymbol{s}_{2}\right), \ldots, T_{f}\left(\boldsymbol{s}_{m}\right)\right\}$ spans $\mathbb{R}_{f}^{m}$. The rest is to prove that $T_{f}(\boldsymbol{s})$ is linearly independent.

Suppose the statement is not true, then there exist $T_{f}(\boldsymbol{s})$ which become linear combination of other members. Let's assume that is $T_{f}\left(\boldsymbol{s}_{\boldsymbol{i}}\right)$ then we have

$$
T^{-1}\left(T_{f}\left(\boldsymbol{s}_{i}\right)\right)=\boldsymbol{s}_{i}=\left(\alpha_{1}\right) \boldsymbol{s}_{1}+\ldots+\left(\alpha_{i-1}\right) \boldsymbol{s}_{i-1}+\left(\alpha_{i+1}\right) \boldsymbol{s}_{i+1}+\ldots+\left(\alpha_{m}\right) \boldsymbol{s}_{m}
$$

But since $\left\{\boldsymbol{s}_{1}, \boldsymbol{s}_{2}, \ldots, \boldsymbol{s}_{\boldsymbol{m}}\right\}$ is linearly independent, then it should be a contradiction.

The above theorem indirectly explains that the internal graph space $\mathbb{R}_{f}^{m}$ has infinitely many vectors that can form a basis for $\mathbb{R}_{f}^{m}$. The next theorems will be discussing about how the basis related each other.

Let $S=\left\{\overrightarrow{s_{1}}, \overrightarrow{s_{2}}, \ldots, \overrightarrow{s_{m}}\right\}$ be a basis for $\mathbb{R}_{f}^{m}$. Then for each $\vec{v} \in \mathbb{R}_{f}^{m}$, one can write

$$
\begin{aligned}
& \vec{v}=\left(\alpha_{1}^{s}\right) \overrightarrow{s_{1}}+\left(\alpha_{2}^{S}\right) \overrightarrow{s_{2}}+\ldots+\left(\alpha_{m}^{s}\right) \overrightarrow{s_{m}} \text { dengan }\left(\alpha_{i}^{s}\right) \in \mathbb{R} . \\
& =\left(\alpha_{1}^{s}\right)\left[\begin{array}{c}
\overrightarrow{s_{1}(1)} \\
\overrightarrow{s_{1}(2)} \\
\vdots \\
\overrightarrow{s_{1}(m)}
\end{array}\right]+\left(\alpha_{2}^{s}\right)\left[\begin{array}{c}
\overrightarrow{s_{2}(1)} \\
\overrightarrow{s_{2}(2)} \\
\vdots \\
\overrightarrow{s_{2}(m)}
\end{array}\right]+\ldots+\left(\alpha_{m}^{s}\right)\left[\begin{array}{c}
\overrightarrow{s_{m}(1)} \\
\overrightarrow{s_{m}(2)} \\
\vdots \\
\overrightarrow{s_{m}(m)}
\end{array}\right]
\end{aligned}
$$

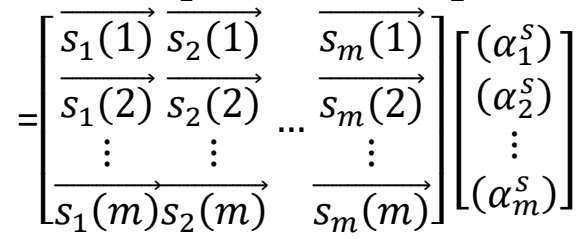

$$
\begin{aligned}
& =[M]_{S, f}[\alpha]_{S}
\end{aligned}
$$

by $[M]_{S, f}$ is matrics which for each entry lies in $\mathbb{R}_{f}^{m}$ and $[\alpha]_{S}$ is real scalar vector.

Then we have

$$
T_{f}^{-1}(\vec{v})=\left[\begin{array}{ccc}
T_{f}^{-1}\left(\overrightarrow{s_{1}(1)}\right) & T_{f}^{-1}\left(\overrightarrow{s_{2}(1)}\right) & T_{f}^{-1}\left(\overrightarrow{s_{m}(1)}\right) \\
T_{f}^{-1}\left(\overrightarrow{s_{1}(2)}\right) & T_{f}^{-1}\left(\overrightarrow{s_{2}(2)}\right) & T_{f}^{-1}\left(\overrightarrow{s_{m}(1)}\right) \\
\vdots & \vdots & \\
T_{f}^{-1}\left(\overrightarrow{s_{1}(m)}\right) T_{f}^{-1}\left(\overrightarrow{s_{2}(m)}\right) & T_{f}^{-1}\left(\overrightarrow{s_{m}(m)}\right)
\end{array}\right]\left[\begin{array}{c}
\left(\alpha_{1}^{s}\right) \\
\left(\alpha_{2}^{s}\right) \\
\vdots \\
\left(\alpha_{m}^{s}\right)
\end{array}\right]
$$


Therefore,

$$
T_{f}^{-1}(\vec{v})=\left[T_{f}^{-1}(S)\right][\alpha]_{S}
$$

The above equation explains how the basis $S$ in internal graph space $\mathbb{R}_{f}^{m}$ mapped to $\mathbb{R}^{m}$. It will make us easier to change basis from the old to the new one. Let's pay attention to the following discussion;

Let $S=\left\{\overrightarrow{s_{1}}, \overrightarrow{s_{2}}, \ldots, \overrightarrow{s_{m}}\right\}$ be a basis of $\mathbb{R}_{f}^{m}$ and $W=\left\{\overrightarrow{w_{1}}, \overrightarrow{w_{2}}, \ldots, \overrightarrow{w_{m}}\right\}$ another basis. We want to change the old basis $S$ into the new one $W$. We have

On the other hand

$$
\vec{v}=[M]_{S, f}[\alpha]_{S}
$$

Therefore

$$
\vec{v}=[M]_{W, f}[\alpha]_{W}
$$

$$
\begin{array}{ll}
T_{f}^{-1}(\vec{v}) & =\left[T_{f}^{-1}(S)\right][\alpha]_{S} \\
\phi_{T_{f}^{-1}(S)} \circ T_{f}^{-1}(\vec{v}) & =[\alpha]_{S}
\end{array}
$$

There exists transition metrics $[M]_{T_{f}^{-1}(S), T_{f}^{-1}(W)}$ such that the following works

$$
\begin{aligned}
\phi_{T_{f}^{-1}(W)} \circ T_{f}^{-1}(\vec{v}) & =[M]_{T_{f}^{-1}(S), T_{f}^{-1}(W)}[\alpha]_{S} \\
& =[\alpha]_{W} .
\end{aligned}
$$

Now we send it back through the map

$$
\phi_{T_{f}^{-1}(W)}^{-1} \circ \phi_{T_{f}^{-1}(W)} \circ T_{f}^{-1}(\vec{v})=\left[T_{f}^{-1}(W)\right][\alpha]_{W} .
$$

Hence

$$
\vec{v}=[M]_{S, f}[\alpha]_{S}=[M]_{W, f}[\alpha]_{W} .
$$

That's how the basis change. For more understanding, let's see the example

Let $f(x)=e^{x}$. The corresponding isomorphism is $T_{f}(x)=\left(x, e^{x}\right)$ for each $x \in \mathbb{R}$.

The graph is $\mathbb{R}_{f}=\left\{a_{f}=\left(a, e^{a}\right): a \in \mathbb{R}\right\}$. By applying the method to find the corresponding addition, we have

$$
a_{f} \oplus b_{f}=\left(a+b, e^{a+b}\right), a, b \in \mathbb{R}
$$

And the scalar multiplication

$$
(\alpha)\left(x_{f}\right)=\left(\alpha x, e^{\alpha x}\right), x \in \mathbb{R}
$$

Now the internal graph space dimension 2 has the form

$$
\mathbb{R}_{f}^{2}=\left\{\left(\begin{array}{c}
x_{f} \\
y_{f}
\end{array}\right): x_{f}, y_{f} \in \mathbb{R}_{f}\right\}
$$

Now let see how the basis change $\mathbb{R}_{f}^{2}$. Choose a basis

and

$$
S=\left\{\left(\begin{array}{c}
\left(2, e^{2}\right) \\
(0,1)
\end{array}\right),\left(\begin{array}{c}
(0,1) \\
\left(2, e^{2}\right)
\end{array}\right)\right\}
$$

$$
W=\left\{\left(\begin{array}{c}
(1, e) \\
(0,1)
\end{array}\right),\left(\begin{array}{c}
(1, e) \\
\left(-1, e^{-1}\right)
\end{array}\right)\right\}
$$

Suppose $\vec{v}=\left(\begin{array}{l}\left(5, e^{5}\right) \\ \left(2, e^{2}\right)\end{array}\right)$. It will be shown that $\vec{v}$ is linear combination of $S$.

Let's pay attention to this

$$
\vec{v}=(\alpha)\left(\begin{array}{c}
\left(2, e^{2}\right) \\
(0,1)
\end{array}\right) \oplus(\beta)\left(\begin{array}{c}
(0,1) \\
\left(2, e^{2}\right)
\end{array}\right)
$$




$$
\begin{aligned}
& =\left(\begin{array}{c}
\left(2 \alpha, e^{2 \alpha}\right) \\
(0,1)
\end{array}\right) \oplus\left(\begin{array}{c}
(0,1) \\
\left(2 \beta, e^{2 \beta}\right)
\end{array}\right) \\
& =\left(\begin{array}{c}
\left(2 \alpha, e^{2 \alpha}\right) \\
\left(2 \beta, e^{2 \beta}\right)
\end{array}\right) \\
& =\left(\begin{array}{c}
\left(5, e^{5}\right) \\
\left(2, e^{2}\right)
\end{array}\right)
\end{aligned}
$$

hence $2 \alpha=5,2 \beta=2, e^{2 \alpha}=e^{5}, e^{2 \beta}=e^{2}$. Those imply $\alpha=\frac{5}{2}$ and $\beta=1$.

Therefore

On the other hand,

$$
\begin{aligned}
\vec{v} & =\left[\begin{array}{ll}
2_{f} & 0_{f} \\
0_{f} & 2_{f}
\end{array}\right]\left[\begin{array}{l}
\frac{5}{2} \\
1
\end{array}\right] \\
& =[M]_{S, f}[\alpha]_{S}
\end{aligned}
$$

$$
\begin{aligned}
\vec{v}= & (\alpha)\left(\begin{array}{c}
(1, e) \\
(0,1)
\end{array}\right) \oplus(\beta)\left(\begin{array}{c}
(1, e) \\
\left(-1, e^{-1}\right)
\end{array}\right) \\
& =\left(\begin{array}{c}
\left(\alpha, e^{\alpha}\right) \\
(0,1)
\end{array}\right) \oplus\left(\begin{array}{c}
\left(\beta, e^{\beta}\right) \\
\left(-\beta, e^{-\beta}\right)
\end{array}\right) \\
& =\left(\begin{array}{c}
\left(\alpha+\beta, e^{\alpha+\beta}\right) \\
\left(-\beta, e^{-\beta}\right)
\end{array}\right) \\
& =\left(\begin{array}{c}
\left(5, e^{5}\right) \\
\left(2, e^{2}\right)
\end{array}\right)
\end{aligned}
$$

We have $\alpha+\beta=5,-\beta=2, e^{\alpha+\beta}=e^{5}, e^{-\beta}=e^{2}$. Those imply $\alpha=7$ and $\beta=-2$.

Therefore

$$
\begin{aligned}
\vec{v} & =\left[\begin{array}{cc}
1_{f} & 1_{f} \\
0_{f} & -1_{f}
\end{array}\right]\left[\begin{array}{c}
7 \\
-2
\end{array}\right] \\
& =[M]_{W, f}[\alpha]_{W} .
\end{aligned}
$$

Its already shown how the vector $\vec{v}$ to be expressed as linear combination of $S$ and $W$. To change basis from $S$ to $W$, we must transfer all members of $S$ to the $\mathbb{R}^{2}$ i.e. $T_{f}^{-1}(S)=$ $\left\{\left(\begin{array}{l}2 \\ 0\end{array}\right),\left(\begin{array}{l}0 \\ 2\end{array}\right)\right\}$ and $T_{f}^{-1}(W)=\left\{\left(\begin{array}{l}1 \\ 1\end{array}\right),\left(\begin{array}{c}0 \\ -1\end{array}\right)\right\}$. By elementary calculation we have

thus, the transition metrics is

$$
\begin{aligned}
& \left(\begin{array}{l}
2 \\
0
\end{array}\right)=2\left(\begin{array}{l}
1 \\
1
\end{array}\right)+2\left(\begin{array}{c}
0 \\
-1
\end{array}\right) \\
& \left(\begin{array}{l}
0 \\
2
\end{array}\right)=0\left(\begin{array}{l}
1 \\
1
\end{array}\right)-2\left(\begin{array}{c}
0 \\
-1
\end{array}\right)
\end{aligned}
$$

$$
\begin{aligned}
\phi_{T_{f}^{-1}(W)} \circ T_{f}^{-1}(\vec{v}) & =\left[\begin{array}{cc}
2 & 0 \\
2 & -2
\end{array}\right]\left[\begin{array}{l}
\frac{5}{2} \\
1
\end{array}\right] \\
& =\left[\begin{array}{c}
7 \\
-2
\end{array}\right] . \text { That's the new coordinate. }
\end{aligned}
$$

\section{CONCLUSIONS}

For each real valued function $f$, the corresponding internal graph space forms a vector space over $\mathbb{R}$. The concept of linear mapping and linear combinations still can be adapted from the graph space and still well defined. 


\section{REFERENCES}

[1] J. Doboš, "On the Set of Points od Discontinuity for Functions with Closed Graphs," vol. 110, no. 1, 1985.

[2] A. V Arhangel, "Relative Topological Properties and Relative Topological Spaces," vol. 8641, no. 95, 1996.

[3] T. Banakh, K. Mine, and K. Sakai, "Classifying homeomorphism groups of infinite graphs," Topol. Appl., vol. 156, no. 17, pp. 2845-2869, 2009.

[4] A. Lazwardi, “Topologi Grafik Fungsi Real Kontinu," in Prosiding Seminar Nasional Pendidikan Matematika, 2017, no. 3185, p. 51.

[5] W. A. Trybulec, "Basis of Vector Space," vol. 2, no. 1, pp. 2-4, 2003.

[6] B. Hou and S. Gao, "The Structure of Some Linear Transformations," Linear Algebra Appl., vol. 437, no. 9, pp. 2110-2116, 2012.

[7] S. Roman, Advanced Lienar Algebra, 3th ed. United States: Springer, 2008.

[8] Y. Zhang, H. Tam, and F. Guo, "Invertible Linear Transformations and the Lie algebras," vol. 13, pp. 682-702, 2008.

[9] S. Waldron, "Frames for Vector Spaces and Affine Spaces," Linear Algebra Appl., vol. 435, no. 1, pp. 77-94, 2011.

[10] A. Aleman, K. Perfekt, S. Richter, and C. Sundberg, "Linear Graph Transformations on Spaces of Analytic," J. Funct. Anal., vol. 268, no. 9, pp. 2707-2734, 2015. 